\title{
Control of Nonlinear Switched Systems Based on Validated Simulation
}

\author{
Adrien Le Coënt ${ }^{\star}$, Julien Alexandre dit Sandretto ${ }^{\mathrm{b}}$, Alexandre Chapoutot ${ }^{\mathrm{b}}$, \\ Laurent Fribourg ${ }^{\mathrm{c}}$ \\ ${ }^{a}$ CMLA, ENS Cachan, CNRS, Université Paris-Saclay, 61 av. du Président Wilson, 94235 Cachan Cedex, France \\ ${ }^{\mathrm{b}}$ U2IS, ENSTA ParisTech, Université Paris-Saclay, 828, Boulevard des Maréchaux, 91762 Palaiseau Cedex, France \\ ${ }^{\mathrm{c}}$ LSV, ENS Cachan, CNRS, Université Paris-Saclay, 61 av. du Président Wilson, 94235 Cachan Cedex, France
}

\begin{abstract}
We present an algorithm of control synthesis for nonlinear switched systems, based on an existing procedure of state-space bisection and made available for nonlinear systems with the help of validated simulation. The use of validated simulation also permits to take bounded perturbations and varying parameters into account. It is particularly interesting for safety critical applications, such as in aeronautical, military or medical fields. The whole approach is entirely guaranteed and the induced controllers are correct-by-design.
\end{abstract}

Key words: Nonlinear control systems, reachability, formal methods, numerical simulation, control system synthesis

\section{Introduction}

We focus here on switched control systems, a class of hybrid systems recently used with success in various domains such as automotive industry and power electronics. These systems are merely described by piecewise dynamics, periodically sampled with a given period. At each period, the system is in one and only one mode, decided by a control rule [14,23]. Moreover, the considered systems can switch between any two modes instantaneously. This simplification can be easily by-passed by the addition of intermediate facticious modes.

In this paper, we consider that these modes are represented by nonlinear ODEs. In order to compute the control of a switched system, we do need the solution of differential equations. In the general case, differential equations can not be integrated formally, and a numerical in-

* This paper was not presented at any IFAC meeting. A short version of this paper appeared in SNR'16 [9]. Corresponding author A. Le Coënt Tel. +33147407429.

Email addresses: adrien.le-coent@ens-cachan.fr (Adrien Le Coënt ${ }^{\star}$ ), alexandre@ensta.fr (Julien Alexandre dit Sandretto), chapoutot@ensta.fr (Alexandre Chapoutot), fribourg@lsv.ens-cachan.fr (Laurent Fribourg). tegration scheme is used to approximate the state of the system. With the objective of computing a guaranteed control, we base our approach on validated simulation (also called "reachability analysis"). The guaranteed or validated solution of ODEs using interval arithmetic is mainly based on two kinds of methods based on: i) Taylor series $[29,30,24,11]$ ii) Runge-Kutta schemes [7,15,6,2]. The former is the oldest method used in interval analysis community because the expression of the bound of a Taylor series is simple to obtain. Nevertheless, the family of Runge-Kutta methods is very important in the field of numerical analysis. Indeed, Runge-Kutta methods have several interesting stability properties which make them suitable for an important class of problems. Our tool [1] implements Runge-Kutta based methods which prove their efficiency at low order for short simulation (fixed by sampling period of controller).

In the methods of symbolic analysis and control of hybrid systems, the way of representing sets of state values and computing reachable sets for systems defined by autonomous ordinary differential equations (ODEs), is fundamental (see, e.g., [16,4]). Many tools using, eg. linearization or hybridization of these dynamics are now available (e.g., Spacex [13], Flow* [8], iSAT-ODE [12]). An interesting approach appeared recently, based on the propagation of reachable sets using guaranteed 
Runge-Kutta methods with adaptive step size control (see $[6,19])$. An originality of the present work is to use such guaranteed integration methods in the framework of switched systems. This notion of guarantee of the results is very interesting, because we are mainly interested into critical domain, such as aeronautical, military and medical ones. Other symbolic approaches for control synthesis of switched systems include the construction of a discrete abstraction of the original system on a grid of the state space. This can be done by computing symbolic models that are approximately bisimilar [17] or approximately alternatingly similar [34] to the original system. Another recent symbolic approach relies on feedback refinement relations [31]. We compare our work with the last two approaches, which are the closest related methods since the associated tools (respectively PESSOA [26] and SCOTS [32]) are used to perform control synthesis on switched systems without any stability assumptions, such as the present method.

The paper is divided as follows. In Section 2, we introduce some preliminaries on switched systems and some notation used in the following. In Section 3, the guaranteed integration of nonlinear ODEs is presented. In Section 4, we present the main algorithm of state-space bisection used for control synthesis. In Section 5, the whole approach is tested on three examples of the literature. We give some performance tests and compare our approach with the state-of-the-art tools in section 6 . We conclude in section 7 .

\section{Switched systems}

Let us consider the nonlinear switched system

$$
\dot{x}(t)=f_{\sigma(t)}(x(t), d(t))
$$

defined for all $t \geq 0$, where $x(t) \in \mathbb{R}^{n}$ is the state of the system, $\sigma(\cdot): \mathbb{R}^{+} \longrightarrow U$ is the switching rule, and $d(t) \in \mathbb{R}^{m}$ is a bounded perturbation. The finite set $U=$ $\{1, \ldots, N\}$ is the set of switching modes of the system. We focus on sampled switched systems: given a sampling period $\tau>0$, switchings will occur at times $\tau, 2 \tau, \ldots$ The switching rule $\sigma(\cdot)$ is thus piecewise constant, we will consider that $\sigma(\cdot)$ is constant on the time interval $[(k-$ 1) $\tau, k \tau)$ for $k \geq 1$. We call "pattern" a finite sequence of modes $\pi=\left(i_{1}, i_{2}, \ldots, i_{k}\right) \in U^{k}$. With such a control input, and under a given perturbation $d$, we will denote by $\mathbf{x}\left(t ; t_{0}, x_{0}, d, \pi\right)$ the solution at time $t$ of the system

$$
\begin{aligned}
\dot{x}(t) & =f_{\sigma(t)}(x(t), d(t)), \\
x\left(t_{0}\right) & =x_{0} \\
\forall j \in\{1, \ldots, k\}, \sigma(t) & =i_{j} \in U \text { for } t \in[(j-1) \tau, j \tau) .
\end{aligned}
$$

We address the problem of synthesizing a statedependent switching rule $\tilde{\sigma}(x)$ for $(2)$ in order to verify some properties. The problem is formalized as follows:
Problem 1 (Control Synthesis Problem) Let us consider a sampled switched system (2). Given three sets $R, S$, and $B$, with $R \cup B \subset S$ and $R \cap B=\emptyset$, find a rule $\tilde{\sigma}(x)$ such that, for any $x(0) \in R$

- $\tau$-stability ${ }^{1}: x(t)$ returns in $R$ infinitely often, at some multiples of sampling time $\tau$.

- safety: $x(t)$ always stays in $S \backslash B$.

Under the above-mentioned notation, we propose a procedure which solves this problem by constructing a law $\tilde{\sigma}(x)$, such that for all $x_{0} \in R$, and under the unknown bounded perturbation $d$, there exists $\pi=\tilde{\sigma}\left(x_{0}\right) \in U^{k}$ for some $k$ such that:

$$
\left\{\begin{array}{r}
\mathbf{x}\left(t_{0}+k \tau ; t_{0}, x_{0}, d, \pi\right) \in R \\
\forall t \in\left[t_{0}, t_{0}+k \tau\right], \quad \mathbf{x}\left(t ; t_{0}, x_{0}, d, \pi\right) \in S \\
\forall t \in\left[t_{0}, t_{0}+k \tau\right], \quad \mathbf{x}\left(t ; t_{0}, x_{0}, d, \pi\right) \notin B
\end{array}\right.
$$

Such a law permits to perform an infinite-time statedependent control. The synthesis algorithm is described in Section 4 and involves guaranteed set based integration presented in the next section, the main underlying tool is interval analysis [29]. To tackle this problem, we introduce some definitions. In the following, we will often use the notation $[x] \in \mathbb{I}$ (the set of intervals with real bounds) where $[x]=[\underline{x}, \bar{x}]=\{x \in \mathbb{R} \mid \underline{x} \leqslant x \leqslant \bar{x}\}$ denotes an interval. By an abuse of notation $[x]$ will also denote a vector of intervals, i.e., a Cartesian product of intervals, a.k.a. a box. In the following, the sets $R, S$ and $B$ are given under the form of boxes.

\section{Definition 1 (Initial Value Problem (IVP)) Consider an ODE with a given initial condition}

$$
\dot{x}(t)=f(t, x(t), d(t)) \quad \text { with } \quad x(0) \in X_{0}, d(t) \in[d],
$$

with $f: \mathbb{R}^{+} \times \mathbb{R}^{n} \times \mathbb{R}^{m} \rightarrow \mathbb{R}^{n}$ assumed to be continuous in $t$ and $d$ and globally Lipschitz in $x$. We assume that parameters d are bounded (used to represent a perturbation, a modeling error, an uncertainty on measurement, ... ). An IVP consists in finding a function $x(t)$ described by the $O D E(3)$ for all $d(t)$ lying in $[d]$ and for all the initial conditions in $X_{0}$.

Definition 2 Let $X \subset \mathbb{R}^{n}$ be a box of the state space. Let $\pi=\left(i_{1}, i_{2}, \ldots, i_{k}\right) \in U^{k}$. The successor set of $X$ via $\pi$, denoted by Post $_{\pi}(X)$, is the (over-approximation of the) image of $X$ induced by application of the pattern $\pi$,

1 This definition of stability is different from the stability in the Lyapunov sense. 
i.e., the solution at time $t=k \tau$ of

$$
\begin{gathered}
\dot{x}(t)=f_{\sigma(t)}(x(t), d(t)), \\
x(0)=x_{0} \in X, \\
\forall t \geq 0, \quad d(t) \in[d], \\
\forall j \in\{1, \ldots, k\}, \quad \sigma(t)=i_{j} \in U \text { for } t \in[(j-1) \tau, j \tau) .
\end{gathered}
$$

Definition 3 Let $X \subset \mathbb{R}^{n}$ be a box of the state space. Let $\pi=\left(i_{1}, i_{2}, \ldots, i_{k}\right) \in U^{k}$. We denote by Tube T $_{\pi}(X)$ the union of boxes covering the trajectories of IVP (4), which construction is detailed in Section 3.

\section{Validated simulation}

In this section, we describe our approach for validated simulation based on Runge-Kutta methods [6,2].

A numerical integration method computes a sequence of approximations $\left(t_{n}, x_{n}\right)$ of the solution $x\left(t ; x_{0}\right)$ of the IVP defined in Equation (3) such that $x_{n} \approx x\left(t_{n} ; x_{n-1}\right)$. The simplest method is Euler's method in which $t_{n+1}=t_{n}+h$ for some step-size $h$ and $x_{n+1}=x_{n}+h \times f\left(t_{n}, x_{n}, d\right)$; so the derivative of $x$ at time $t_{n}, f\left(t_{n}, x_{n}, d\right)$, is used as an approximation of the derivative on the whole time interval to perform a linear interpolation. This method is very simple and fast, but requires small step-sizes. More advanced methods coming from the Runge-Kutta family use a few intermediate computations to improve the approximation of the derivative. The general form of an explicit $s$-stage Runge-Kutta formula, that is using $s$ evaluations of $f$, is

$$
\begin{array}{r}
x_{n+1}=x_{n}+h \sum_{i=1}^{s} b_{i} k_{i}, \\
k_{1}=f\left(t_{n}, x_{n}, d\right), \\
k_{i}=f\left(t_{n}+c_{i} h, x_{n}+h \sum_{j=1}^{i-1} a_{i j} k_{j}, d\right), i=2,3, \ldots, s .
\end{array}
$$

The coefficients $c_{i}, a_{i j}$ and $b_{i}$ fully characterize the method. To make Runge-Kutta validated, the challenging question is how to compute a bound on the distance between the true solution and the numerical solution, defined by $x\left(t_{n} ; x_{n-1}\right)-x_{n}$. This distance is associated to the local truncation error (LTE) of the numerical method.

To bound the LTE, we rely on order condition [18] respected by all Runge-Kutta methods. This condition states that a method of this family is of order $p$ iff the $p+1$ first coefficients of the Taylor expansion of the solution and the Taylor expansion of the numerical methods are equal. In consequence, LTE is proportional to the Lagrange remainders of Taylor expansions. Formally, LTE is defined by (see [6]):

$$
\begin{aligned}
& x\left(t_{n} ; x_{n-1}\right)-x_{n}= \\
& \frac{h^{p+1}}{(p+1) !}\left(\begin{array}{c}
\left.f^{(p)}\left(\xi, x\left(\xi ; x_{n-1}\right), d\right)-\frac{d^{p+1} \phi}{d t^{p+1}}(\eta)\right) \\
\xi \in] t_{n}, t_{n+1}[\text { and } \eta \in] t_{n}, t_{n+1}[.
\end{array}\right.
\end{aligned}
$$

The function $f^{(n)}$ stands for the $n$-th derivative of function $f$ w.r.t. time $t$ that is $\frac{d^{n} f}{d t^{n}}$ and $h=t_{n+1}-t_{n}$ is the step-size. The function $\phi: \mathbb{R} \rightarrow \mathbb{R}^{n}$ is defined by $\phi(t)=x_{n}+h \sum_{i=1}^{s} b_{i} k_{i}(t)$ where $k_{i}(t)$ are defined as Equation (5).

The challenge to make Runge-Kutta integration schemes safe w.r.t. the true solution of IVP is then to compute a bound of the result of Equation (6). In other words we have to bound the value of $f^{(p)}\left(\xi, x\left(\xi ; x_{n-1}\right), d\right)$ and the value of $\frac{d^{p+1} \phi}{d t^{p+1}}(\eta)$. The latter expression is straightforward to bound because the function $\phi$ only depends on the value of the step-size $h$, and so does its $(p+1)$ th derivative. The bound is then obtain using the affine arithmetic $[10,3]$.

However, the expression $f^{(p)}\left(\xi, x\left(\xi ; x_{n-1}\right), d\right)$ is not so easy to bound as it requires to evaluate $f$ for a particular value of the IVP solution $x\left(\xi ; x_{n-1}\right)$ at an unknown time $\xi \in] t_{n}, t_{n+1}[$. The solution used is the same as the one found in $[30,7]$ and it requires to bound the solution of IVP on the interval $\left[t_{n}, t_{n+1}\right]$. This bound is usually computed using the Banach's fixpoint theorem applied with the Picard-Lindelöf operator, see [30]. This operator is used to compute an enclosure of the solution $[\tilde{x}]$ of IVP over a time interval $\left[t_{n}, t_{n+1}\right]$, that is for all $t \in\left[t_{n}, t_{n+1}\right], x\left(t ; x_{n-1}\right) \in[\tilde{x}]$. We can hence bound $f^{(p)}$ substituting $x\left(\xi ; x_{n-1}\right)$ by $[\tilde{x}]$.

For a given pattern of switched modes $\pi=\left(i_{1}, \ldots, i_{k}\right) \in$ $U^{k}$ of length $k$, we are able to compute, for $j \in\{1, . ., k\}$, the enclosures:

- $\left[x_{j}\right] \ni x\left(t_{j}\right)$;

- $\left[\tilde{x}_{j}\right] \ni x(t)$, for $t \in[(j-1) \tau, j \tau]$.

with respect to the system of IVPs:

$$
\left\{\begin{array}{c}
\dot{x}(t)=f_{\sigma(t)}(t, x(t), d(t)), \\
x\left(t_{0}=0\right) \in\left[x_{0}\right], d(t) \in[d], \\
\sigma(t)=i_{1}, \forall t \in\left[0, t_{1}\right], t_{1}=\tau \\
\vdots \\
\dot{x}(t)=f_{\sigma(t)}(t, x(t), d(t)), \\
x\left(t_{k-1}\right) \in\left[x_{k-1}\right], d(t) \in[d], \\
\sigma(t)=i_{k}, \forall t \in\left[t_{k-1}, t_{k}\right], t_{k}=k \tau
\end{array}\right.
$$


Thereby, the enclosure $\operatorname{Post}_{\pi}\left(\left[x_{0}\right]\right)$ is included in $\left[x_{k}\right]$ and Tube $_{\pi}\left(\left[x_{0}\right]\right)$ is included in $\bigcup_{j=1, . ., k}\left[\tilde{x}_{j}\right]$. This applies for all initial states in $\left[x_{0}\right]$ and all disturbances $d(t) \in[d]$. A view of enclosures computed by the validated simulation for one solution obtained for Example 5.2 is shown in Figure 1.

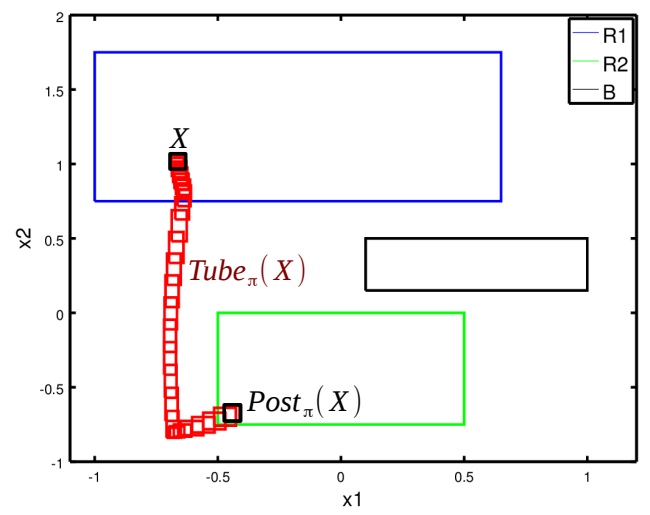

Fig. 1. Functions $\operatorname{Post}_{\pi}(X)$ and $T u b e_{\pi}(X)$ for the initial box $X=[-0.69,-0.64] \times[1,1.06]$, with a pattern $\pi=(1,3,0)$.

\section{The state-space bisection algorithm}

\subsection{Principle of the algorithm}

We describe here the algorithm solving the control synthesis problem (see Problem 1, Section 2). Given the input boxes $R, S, B$, and given two positive integers $K$ and $D$, the algorithm provides, when it succeeds, a decomposition $\Delta$ of $R$ of the form $\left\{V_{i}, \pi_{i}\right\}_{i \in I}$, with the properties:

$\bigcup_{i \in I} V_{i}=R$

$\forall i \in I, \operatorname{Post}_{\pi_{i}}\left(V_{i}\right) \subseteq R$,

$\forall i \in I, T u b e_{\pi_{i}}\left(V_{i}\right) \subseteq S$,

$\forall i \in I$, Tube $_{\pi_{i}}\left(V_{i}\right) \bigcap B=\emptyset$.

The sub-boxes $\left\{V_{i}\right\}_{i \in I}$ are obtained by repeated bisection. At first, function Decomposition calls sub-function Find_Pattern which looks for a pattern $\pi$ of length at most $K$ such that $\operatorname{Post}_{\pi}(R) \subseteq R$, Tube $_{\pi}(R) \subseteq S$ and Tube $_{\pi}(R) \bigcap B=\emptyset$. If such a pattern $\pi$ is found, then a uniform control over $R$ is found (see Figure 2(a)). Otherwise, $R$ is divided into two sub-boxes $V_{1}, V_{2}$, by bisecting $R$ w.r.t. its longest dimension. Patterns are then searched to control these sub-boxes (see Figure 2(b)). If for each $V_{i}$, function Find_Pattern manages to get a pattern $\pi_{i}$ of length at most $K$ verifying Post $_{\pi_{i}}\left(V_{i}\right) \subseteq R$, Tube $_{\pi_{i}}\left(V_{i}\right) \subseteq S$ and Tube $_{\pi_{i}}\left(V_{i}\right) \cap B=\emptyset$, then it is done. If, for some $V_{j}$, no such pattern is found, the procedure is recursively applied to $V_{j}$. It ends with success when every sub-box of $R$ has a pattern verifying the latter conditions, or fails when the maximal degree of decomposition $D$ is reached. The algorithmic form of functions Decomposition and Find_Pattern is given in Figures 3 (cf. 4 and in $[14,20]$ for the linear case).
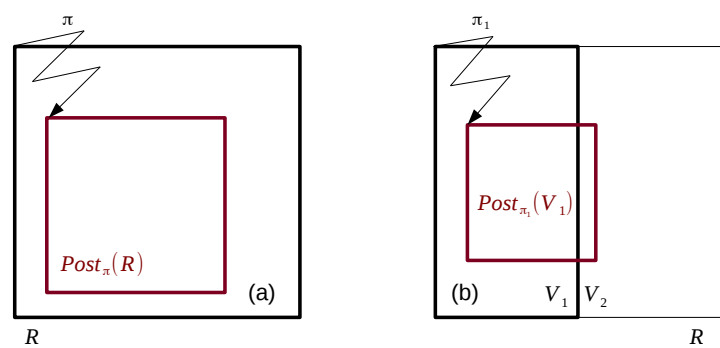

Fig. 2. Principle of the bisection method.

Having defined the control synthesis method, we now introduce the main result of this paper, stated as follows:

Proposition 1 The algorithm of Figure 3 with input $(R, R, S, B, D, K)$ outputs, when it successfully terminates, a decomposition $\left\{V_{i}, \pi_{i}\right\}_{i \in I}$ of $R$ which solves Problem 1.

Proof 1 Let $x_{0}=x\left(t_{0}=0\right)$ be an initial condition belonging to $R$. If the decomposition has terminated successfully, we have $\bigcup_{i \in I} V_{i}=R$, and $x_{0}$ thus belongs to $V_{i_{0}}$ for some $i_{0} \in I$. We can thus apply the pattern $\pi_{i_{0}}$ associated to $V_{i_{0}}$. Let us denote by $k_{0}$ the length of $\pi_{i_{0}}$. We have:

- $\mathbf{x}\left(k_{0} \tau ; 0, x_{0}, d, \pi_{i_{0}}\right) \in R$

- $\forall t \in\left[0, k_{0} \tau\right], \quad \mathbf{x}\left(t ; 0, x_{0}, d, \pi_{i_{0}}\right) \in S$

- $\forall t \in\left[0, k_{0} \tau\right], \quad \mathbf{x}\left(t ; 0, x_{0}, d, \pi_{i_{0}}\right) \notin B$

Let $x_{1}=\mathbf{x}\left(k_{0} \tau ; 0, x_{0}, d, \pi_{i_{0}}\right) \in R$ be the state reached after application of $\pi_{i_{0}}$ and let $t_{1}=k_{0} \tau$. State $x_{1}$ belongs to $R$, it thus belongs to $V_{i_{1}}$ for some $i_{1} \in I$, and we can apply the associated pattern $\pi_{i_{1}}$ of length $k_{1}$, leading to:

- $\mathbf{x}\left(t_{1}+k_{1} \tau ; t_{1}, x_{1}, d, \pi_{i_{1}}\right) \in R$

- $\forall t \in\left[t_{1}, t_{1}+k_{1} \tau\right], \quad \mathbf{x}\left(t ; t_{1}, x_{1}, d, \pi_{i_{1}}\right) \in S$

- $\forall t \in\left[t_{1}, t_{1}+k_{1} \tau\right], \quad \mathbf{x}\left(t ; t_{1}, x_{1}, d, \pi_{i_{1}}\right) \notin B$

We can then iterate this procedure from the new state $x_{2}=\mathbf{x}\left(t_{1}+k_{1} \tau ; t_{1}, x_{1}, d, \pi_{i_{1}}\right) \in R$. This can be repeated infinitely, yielding a sequence of points belonging to $R$ $x_{0}, x_{1}, x_{2}, \ldots$ attained at times $t_{0}, t_{1}, t_{2}, \ldots$, at which the patterns $\pi_{i_{0}}, \pi_{i_{1}}, \pi_{i_{2}}, \ldots$ are applied.

We furthermore have that all the trajectories stay in $S$ and never cross $B: \forall t \in \mathbb{R}^{+}, \exists k \geq 0, t \in$ $\left[t_{k}, t_{k+1}\right]$ and $\forall t \in\left[t_{k}, t_{k+1}\right], \mathbf{x}\left(t ; t_{k}, x_{k}, d, \pi_{i_{k}}\right) \in$ $S, \mathbf{x}\left(t ; t_{k}, x_{k}, d, \pi_{i_{k}}\right) \notin B$. The trajectories thus return infinitely often in $R$, while always staying in $S$ and never crossing $B$. 
Function: Decomposition $(W, R, S, B, D, K)$

Input: A box $W$, a box $R$, a box $S$, a box $B$, a degree $D$ of bisection, a length $K$ of input pattern

Output: $\left\langle\left\{\left(V_{i}, \pi_{i}\right)\right\}_{i}\right.$, True $\rangle$ or $\left\langle_{-}\right.$, False $\rangle$

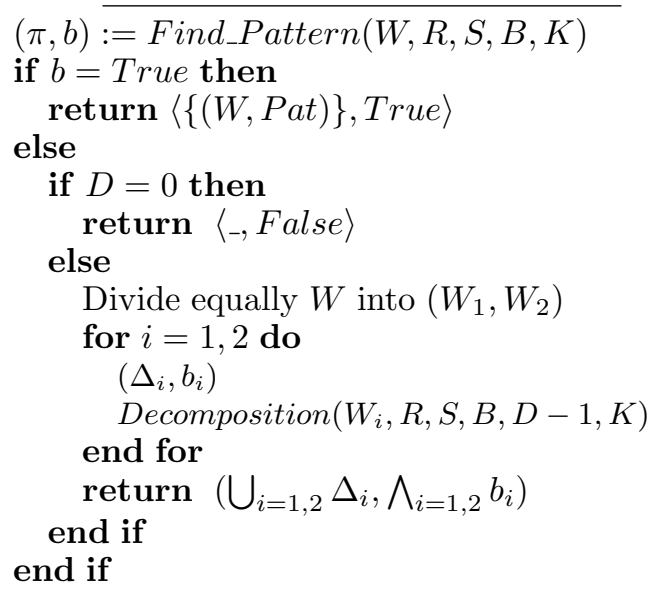

Fig. 3. Algorithmic form of Function Decomposition.

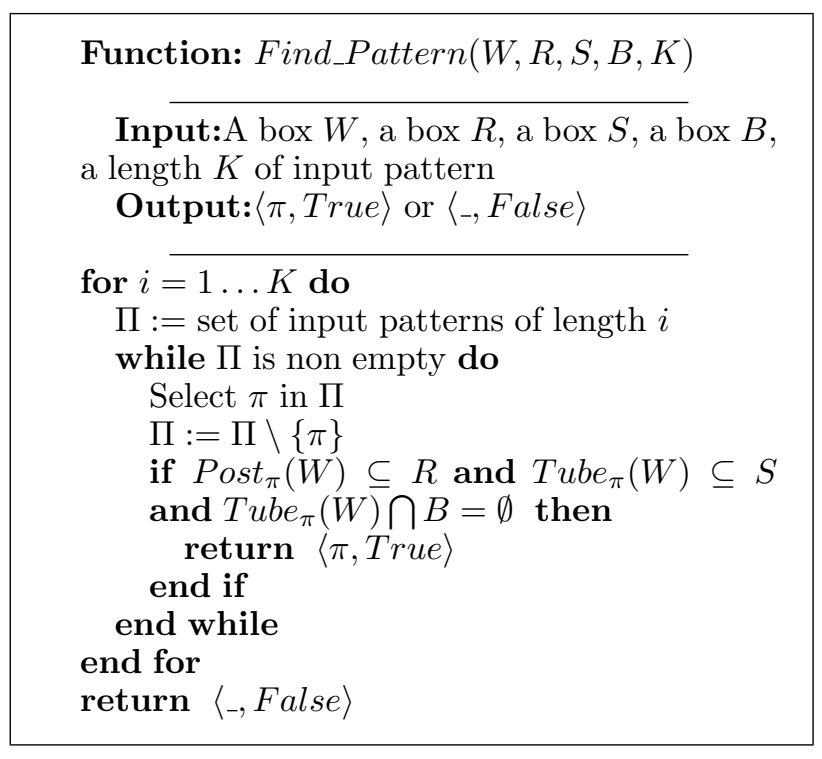

Fig. 4. Algorithmic form of Function Find_Pattern.

Remark 1 Note that it is possible to perform reachability from a set $R_{1}$ to another set $R_{2}$ by computing Decomposition $\left(R_{1}, R_{2}, S, B, D, K\right)$. The set $R_{1}$ is thus decomposed with the objective to send its sub-boxes into $R_{2}$, i.e. for a sub-box $V$ of $R_{1}$, patterns $\pi$ are searched with the objective Post $(V) \subseteq R_{2}$ (see Example 5.2).

\subsection{The research of patterns}

We propose here an improvement of the function Find_Pattern given in $[9,14,20]$, which is a naive testing of all the patterns of growing length (up to $K$ ).

The improved function, denoted here by Find_Pattern2, exploits heuristics to prune the search tree of patterns. The algorithmic form of Find_Pattern 2 is given in Figure 5. It relies on a new data structure consisting of a list of triplets containing:

- An initial box $V \subset \mathbb{R}^{n}$,

- A current box $\operatorname{Post}_{\pi}(V)$, image of $V$ by the pattern $\pi$,

- The associated pattern $\pi$.

For any element $e$ of a list of this type, we denote by e. $Y_{\text {init }}$ the initial box, e. $Y_{\text {current }}$ the current box, and by $e . \Pi$ the associated pattern. We denote by $e_{\text {current }}=$ takeHead $(\mathcal{L})$ the element on top of a list $\mathcal{L}$ (this element is removed from list $\mathcal{L})$. The function $\operatorname{putTail}(\cdot, \mathcal{L})$ adds an element at the end of the list $\mathcal{L}$.

Let us suppose one wants to control a box $X \subseteq R$. The list $\mathcal{L}$ of Figure 5 is used to store the intermediate computations leading to possible solutions (patterns sending $X$ in $R$ while never crossing $B$ or $\mathbb{R}^{n} \backslash S$ ). It is initialized as $\mathcal{L}=\{(X, X, \emptyset)\}$. First, a testing of all the control modes is performed (a set simulation starting from $W$ during time $\tau$ is computed for all the modes in $U$ ). The first level of branches is thus tested exhaustively. If a branch leads to crossing $B$ or $\mathbb{R}^{n} \backslash S$, the branch is cut. Otherwise, either a solution is found or an intermediate state is added to $\mathcal{L}$. The next level of branches (patterns of length 2) is then explored from branches that are not cut. And so on iteratively. At the end, either the tree is explored up to level $K$ (avoiding the cut branches), or all the branches have been cut at lower levels. List $\mathcal{L}$ is thus of the form $\left\{\left(X, \text { Post }_{\pi_{i}}(X), \pi_{i}\right)\right\}_{i \in I_{X}}$, where for each $i \in I_{X}$ we have Post $_{\pi_{i}}(X) \subseteq S$ and Tube $\pi_{i}(X) \cap B=\emptyset$. Here, $I_{X}$ is the set of indexes associated to the stored intermediate solutions, $\left|I_{X}\right|$ is thus the number of stored intermediate solutions for the initial box $X$. The number of stored intermediate solutions grows as the search tree of patterns is explored, then decreases as solutions are validated, branches are cut, or the maximal level $K$ is reached.

The storage of the intermediate solutions $\operatorname{Post}_{\pi_{i}}(X)$ allows to reuse the computations already performed. Even if the search tree of patterns is visited exhaustively, it already allows to obtain much better computation times than with Function Find_Pattern.

A second list, denoted by Solution in Figure 5, is used to store the validated patterns associated to $X$, i.e. a list of patterns of the form $\left\{\pi_{j}\right\}_{j \in I_{X}^{\prime}}$, where for each $j \in I_{X}^{\prime}$ we have Post $_{\pi_{j}}(X) \subseteq R$, Tube $\pi_{j}(X) \cap B=$ $\emptyset$ and Tube $_{\pi_{j}}(X) \subseteq S$. Here, $I_{X}^{\prime}$ is the set of indexes associated the the stored validated solutions, $\left|I_{X}^{\prime}\right|$ is thus the number of stored validated solutions for the initial box $X$. The number of stored validated solutions can only increase, and we hope that at least one solution is 
found, otherwise, the initial box $X$ is split in two subboxes.

Note that several solutions can be returned by Find_Pattern2, further optimizations could thus be performed, such as returning the pattern minimizing a given cost function. In practice, and in the examples given below, we return the first validated pattern and stop the computation as soon as it is obtained (see commented line in Figure 5).

Compared to $[14,20]$, this new function highly improves the computation times, even though the complexity of the two functions is theoretically the same, at most in $O\left(N^{K}\right)$. A comparison between functions Find_Pattern and Find_Pattern 2 is given in Section 6 .

\section{$5 \quad$ Experimentations}

In this section, we apply our approach to different case studies taken from the literature. Our solver prototype is written in $\mathrm{C}++$ and based on DynIBEX [1]. The computations times given in the following have been performed on a $2.80 \mathrm{GHz}$ Intel Core i7-4810MQ CPU with $8 \mathrm{~GB}$ of memory. Note that our algorithm is mono-threaded so all the experimentation only uses one core to perform the computations. The results given in this section have been obtained with Function Find_Pattern2.

\subsection{A linear example: boost DC-DC converter}

This linear example is taken from [5] and has already been treated with the state-space bisection method in a linear framework in [14].

The system is a boost DC-DC converter with one switching cell. There are two switching modes depending on the position of the switching cell. The dynamics is given by the equation $\dot{x}(t)=A_{\sigma(t)} x(t)+B_{\sigma(t)}$ with $\sigma(t) \in$ $U=\{1,2\}$. The two modes are given by the matrices:

$$
\begin{gathered}
A_{1}=\left(\begin{array}{cc}
-\frac{r_{l}}{x_{l}} & 0 \\
0 & -\frac{1}{x_{c}} \frac{1}{r_{0}+r_{c}}
\end{array}\right) \quad B_{1}=\left(\begin{array}{c}
\frac{v_{s}}{x_{l}} \\
0
\end{array}\right) \\
A_{2}=\left(\begin{array}{cc}
-\frac{1}{x_{l}}\left(r_{l}+\frac{r_{0} \cdot r_{c}}{r_{0}+r_{c}}\right) & -\frac{1}{x_{l}} \frac{r_{0}}{r_{0}+r_{c}} \\
\frac{1}{x_{c}} \frac{r_{0}}{r_{0}+r_{c}} & -\frac{1}{x_{c}} \frac{r_{0}}{r_{0}+r_{c}}
\end{array}\right) \quad B_{2}=\left(\begin{array}{c}
\frac{v_{s}}{x_{l}} \\
0
\end{array}\right)
\end{gathered}
$$

with $x_{c}=70, x_{l}=3, r_{c}=0.005, r_{l}=0.05, r_{0}=1$, $v_{s}=1$. The sampling period is $\tau=0.5$. The parameters are exact and there is no perturbation. We want the state to return infinitely often to the region $R$, set here to $[1.55,2.15] \times[1.0,1.4]$, while never going out of the safety set $S=[1.54,2.16] \times[0.99,1.41]$.

The decomposition was obtained in less than one second with a maximum length of pattern set to $K=6$ and a maximum bisection depth of $D=3$. A simulation is given in Figure 6.
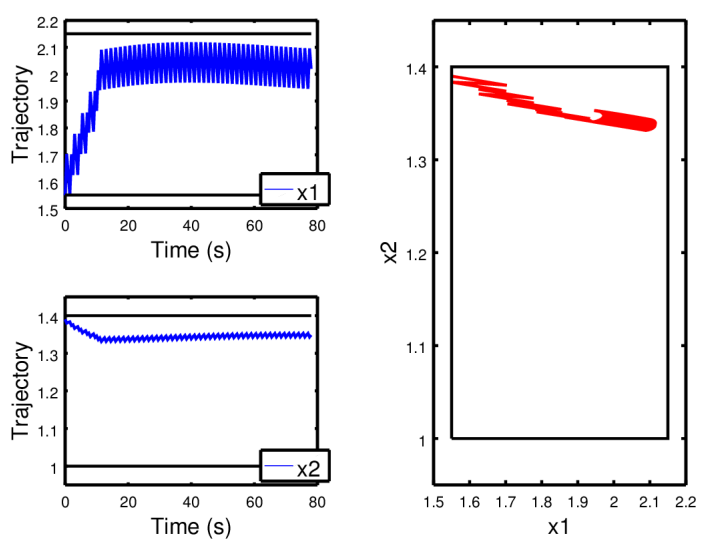

Fig. 6. Simulation from the initial condition $(1.55,1.4)$. The box $R$ is in plain black. The trajectory is plotted within time for the two state variables on the left, and in the state-space plane on the right.

\subsection{A polynomial example}

We consider the polynomial system taken from [25]:

$$
\left[\begin{array}{c}
\dot{x}_{1} \\
\dot{x}_{2}
\end{array}\right]=\left[\begin{array}{c}
-x_{2}-1.5 x_{1}-0.5 x_{1}^{3}+u_{1}+d_{1} \\
x_{1}+u_{2}+d_{2}
\end{array}\right] .
$$

The control inputs are given by $u=\left(u_{1}, u_{2}\right)=$ $K_{\sigma(t)}\left(x_{1}, x_{2}\right), \sigma(t) \in U=\{1,2,3,4\}$, which correspond to four different state feedback controllers $K_{1}(x)=\left(0,-x_{2}^{2}+2\right), K_{2}(x)=\left(0,-x_{2}\right), K_{3}(x)=$ $(2,10), K_{4}(x)=(-1.5,10)$. We thus have four switching modes. The disturbance $d=\left(d_{1}, d_{2}\right)$ lies in $[-0.005,0.005] \times[-0.005,0.005]$. The objective is to visit infinitely often two zones $R_{1}$ and $R_{2}$, without going out of a safety zone $S$, and while never crossing a forbidden zone $B$. Two decompositions are performed:

- a decomposition of $R_{1}$ which returns $\left\{\left(V_{i}, \pi_{i}\right)\right\}_{i \in I_{1}}$ with:

$$
\begin{aligned}
& \bigcup_{i \in I_{1}} V_{i}=R_{1}, \\
& \forall i \in I_{1}, \text { Post }_{\pi_{i}}\left(V_{i}\right) \subseteq R_{2}, \\
& \forall i \in I_{1}, \text { Tube }_{\pi_{i}}\left(V_{i}\right) \subseteq S, \\
& \forall i \in I_{1}, \text { Tube }_{\pi_{i}}\left(V_{i}\right) \bigcap B=\emptyset .
\end{aligned}
$$

- a decomposition of $R_{2}$ which returns $\left\{\left(V_{i}, \pi_{i}\right)\right\}_{i \in I_{2}}$ with:

$$
\bigcup_{i \in I_{2}} V_{i}=R_{2}
$$


Function: Find_Pattern $2(W, R, S, B, K)$

Input:A box $W$, a box $R$, a box $S$, a box $B$, a length $K$ of input pattern Output: $\langle\pi$, True $\rangle$ or $\left\langle_{-}\right.$, False $\rangle$

Solution $=\{\emptyset\}$

$\mathcal{L}=\{(W, W, \emptyset)\}$

while $\mathcal{L} \neq \emptyset$ do

$e_{\text {current }}=\operatorname{takeHead}(\mathcal{L})$

for $i \in U$ do

if Post $_{i}\left(e_{\text {current }} . Y_{\text {current }}\right) \subseteq R$ and Tube $_{i}\left(e_{\text {current }} . Y_{\text {current }}\right) \cap B=\emptyset$ and

Tube
$\quad\left(e_{\text {current }} . Y_{\text {current }}\right) \subseteq S$ then
putTail $\left(\right.$ Solution, $\left.e_{\text {current }} . \Pi+i\right) \quad / *$ can be replaced by: "return $\left\langle e_{\text {current }} . \Pi+i\right.$, True $\rangle$ " */ else

if Tube $_{i}\left(e_{\text {current }} . Y_{\text {current }}\right) \cap B \neq \emptyset$ or Tube $e_{i}\left(e_{\text {current }} . Y_{\text {current }}\right) \nsubseteq \subseteq$ then

discard $e_{\text {current }}$

end if

else

if Tube $e_{i}\left(e_{\text {current }} . Y_{\text {current }}\right) \bigcap B=\emptyset$ and Tube $e_{i}\left(e_{\text {current }} . Y_{\text {current }}\right) \subseteq S$ then

if Length $(\Pi)+1<K$ then

$\operatorname{putTail}\left(\mathcal{L},\left(e_{\text {current }} . Y_{\text {init }}\right.\right.$, Post $\left.\left._{i}\left(e_{\text {current }} . Y_{\text {current }}\right), e_{\text {current }} . \Pi+i\right)\right)$

end if

end if

end if

end for

end while

return $\left\langle_{-}\right.$, False $\rangle$if no solution is found, or $\langle\pi$, True $\rangle, \pi$ being any pattern validated in Solution.

Fig. 5. Algorithmic form of Function Find_Pattern2.

$\forall i \in I_{2}$, Post $_{\pi_{i}}\left(V_{i}\right) \subseteq R_{1}$,

$\forall i \in I_{2}$, Tube $_{\pi_{i}}\left(V_{i}\right) \subseteq S$

$\forall i \in I_{2}$, Tube $_{\pi_{i}}\left(V_{i}\right) \bar{\bigcap} B=\emptyset$.

The input boxes are the following:

$R_{1}=[-0.5,0.5] \times[-0.75,0.0]$,

$R_{2}=[-1.0,0.65] \times[0.75,1.75]$,

$S=[-2.0,2.0] \times[-1.5,3.0]$,

$B=[0.1,1.0] \times[0.15,0.5]$.

The sampling period is set to $\tau=0.15$. The decompositions were obtained in 2 minutes and 30 seconds with a maximum length of pattern set to $K=12$ and a maximum bisection depth of $D=5$. A simulation is given in Figure 7 in which the disturbance $d$ is chosen randomly in $[-0.005,0.005] \times[-0.005,0.005]$ at every time step.

\subsection{Building ventilation}

We consider a building ventilation application adapted from [27]. The system is a four room apartment subject to heat transfer between the rooms, with the external
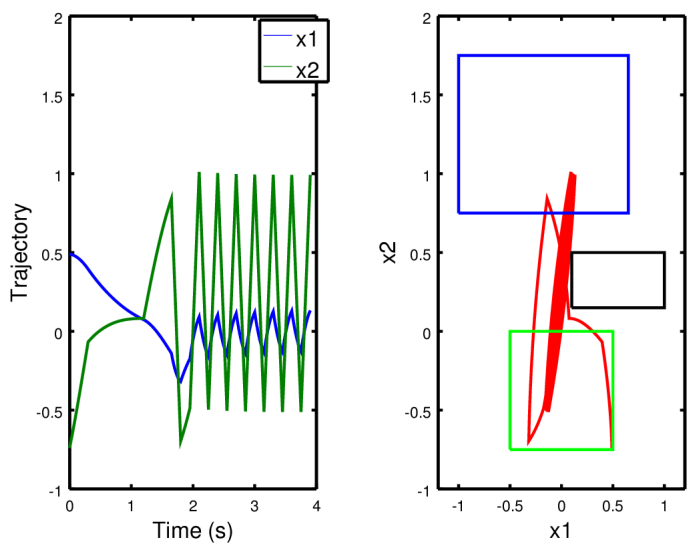

Fig. 7. Simulation from the initial condition $(0.5,-0.75)$. The trajectory is plotted within time on the left, and in the state space plane on the right. In the sate space plane, the set $R_{1}$ is in plain green, $R_{2}$ in plain blue, and $B$ in plain black.

environment, with the underfloor, and with human beings. The dynamics of the system is given by the follow- 
ing equation:

$$
\begin{aligned}
\frac{d T_{i}}{d t}=\sum_{j \in \mathcal{N}^{*}} a_{i j} & \left(T_{j}-T_{i}\right)+\delta_{s_{i}} b_{i}\left(T_{s_{i}}^{4}-T_{i}^{4}\right) \\
& +c_{i} \max \left(0, \frac{V_{i}-V_{i}^{*}}{\bar{V}_{i}-V_{i}^{*}}\right)\left(T_{u}-T_{i}\right)
\end{aligned}
$$

The state of the system is given by the temperatures in the rooms $T_{i}$, for $i \in \mathcal{N}=\{1, \ldots, 4\}$. Room $i$ is subject to heat exchange with different entities stated by the indexes $\mathcal{N}^{*}=\{1,2,3,4, u, o, c\}$.

The heat transfer between the rooms is given by the coefficients $a_{i j}$ for $i, j \in \mathcal{N}^{2}$, and the different perturbations are the following:

- The external environment: it has an effect on room $i$ with the coefficient $a_{i o}$ and the outside temperature $T_{o}$, varying between $27^{\circ} \mathrm{C}$ and $30^{\circ} \mathrm{C}$.

- The heat transfer through the ceiling: it has an effect on room $i$ with the coefficient $a_{i c}$ and the ceiling temperature $T_{c}$, varying between $27^{\circ} \mathrm{C}$ and $30^{\circ} \mathrm{C}$.

- The heat transfer with the underfloor: it is given by the coefficient $a_{i u}$ and the underfloor temperature $T_{u}$, set to $17^{\circ} \mathrm{C}\left(T_{u}\right.$ is constant, regulated by a PID controller).

- The perturbation induced by the presence of humans: it is given in room $i$ by the term $\delta_{s_{i}} b_{i}\left(T_{s_{i}}^{4}-T_{i}^{4}\right)$, the parameter $\delta_{s_{i}}$ is equal to 1 when someone is present in room $i, 0$ otherwise, and $T_{s_{i}}$ is a given identified parameter.

The control $V_{i}, i \in \mathcal{N}$, is applied through the term $c_{i} \max \left(0, \frac{V_{i}-V_{i}^{*}}{\bar{V}_{i}-V_{i}^{*}}\right)\left(T_{u}-T_{i}\right)$. A voltage $V_{i}$ is applied to force ventilation from the underfloor to room $i$, and the command of an underfloor fan is subject to a dry friction. Because we work in a switched control framework, $V_{i}$ can take only discrete values, which removes the problem of dealing with a "max" function in interval analysis. In the experiment, $V_{1}$ and $V_{4}$ can take the values $0 \mathrm{~V}$ or $3.5 \mathrm{~V}$, and $V_{2}$ and $V_{3}$ can take the values $0 \mathrm{~V}$ or $3 \mathrm{~V}$. This leads to a system of the form (1) with $\sigma(t) \in U=\{1, \ldots, 16\}$, the 16 switching modes corresponding to the different possible combinations of voltages $V_{i}$. The sampling period is $\tau=10$ s.

The parameters $T_{s_{i}}, V_{i}^{*}, \bar{V}_{i}, a_{i j}, b_{i}, c_{i}$ are given in [27] and have been identified with a proper identification procedure detailed in [28]. Note that here we have neglected the term $\sum_{j \in \mathcal{N}} \delta_{d_{i j}} c_{i, j} * h\left(T_{j}-T_{i}\right)$ of [27], representing the perturbation induced by the open or closed state of the doors between the rooms. Taking a "max" function into account with interval analysis is actually still a difficult task. However, this term could have been taken into account with a proper regularization (smoothing).
The decomposition was obtained in 4 minutes with a maximum length of pattern set to $K=2$ and a maximum bisection depth of $D=4$. The perturbation due to human beings has been taken into account by setting the parameters $\delta_{s_{i}}$ equal to the whole interval $[0,1]$ for the decomposition, and the imposed perturbation for the simulation is given Figure 8. The temperatures $T_{o}$ and $T_{c}$ have been set to the interval $[27,30]$ for the decomposition, and are set to $30^{\circ} \mathrm{C}$ for the simulation. A simulation of the controller obtained with the state-space bisection procedure is given in Figure 9, where the control objective is to stabilize the temperature in $[20,22]^{4}$ while never going out of $[19,23]^{4}$.
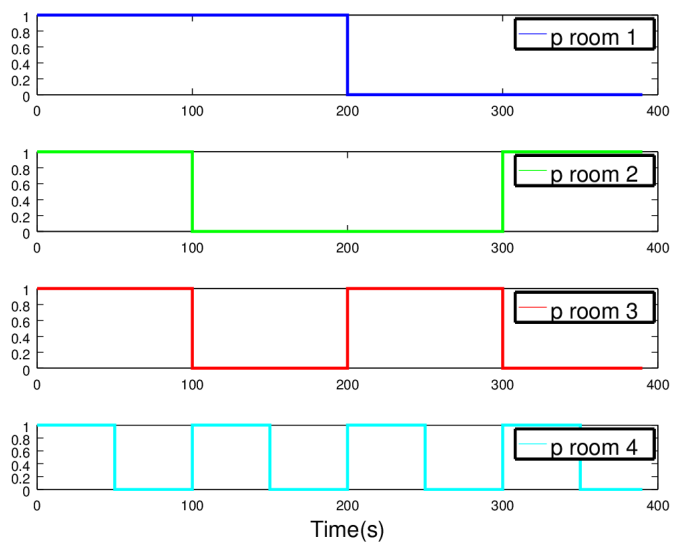

Fig. 8. Perturbation (presence of humans) imposed within time in the different rooms.

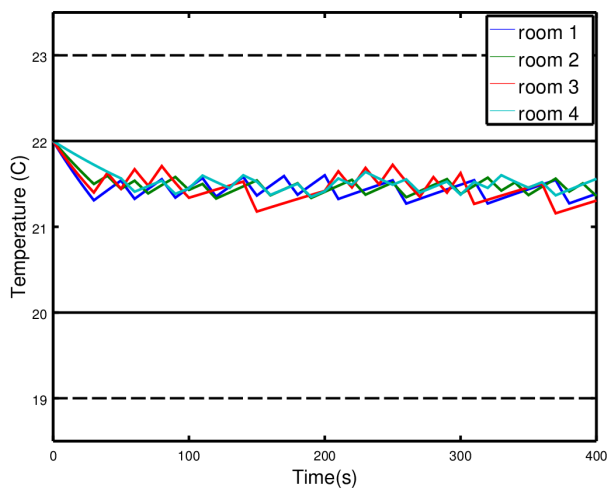

Fig. 9. Simulation from the initial condition $(22,22,22,22)$. The objective set $R$ is in plain black and the safety set $S$ is in dotted black.

\section{Performance tests}

We present a comparison of the computation times obtained with functions Find_Pattern, Find_Pattern2, and with the state-of-the-art tools PESSOA [26] and SCOTS [32]. 
Table 1

Comparison of Find_Pattern and Find_Pattern2.

\begin{tabular}{|c|c|c|}
\hline \multirow{2}{*}{ Example } & \multicolumn{2}{|c|}{ Computation time } \\
\cline { 2 - 3 } & Find_Pattern & Find_Pattern2 \\
\hline DC-DC Converter & $1609 \mathrm{~s}$ & $<1 \mathrm{~s}$ \\
Polynomial example & Time Out & $150 \mathrm{~s}$ \\
Building ventilation & $272 \mathrm{~s}$ & $228 \mathrm{~s}$ \\
\hline
\end{tabular}

Table 2

Comparison with state-of-the-art tools.

\begin{tabular}{|c|c|c|c|}
\hline \multirow{2}{*}{ Example } & \multicolumn{3}{|c|}{ Computation time } \\
\cline { 2 - 4 } & FP2 & SCOTS & PESSOA \\
\hline DC-DC Converter & $<1 \mathrm{~s}$ & $43 \mathrm{~s}$ & $760 \mathrm{~s}$ \\
Polynomial example & $150 \mathrm{~s}$ & $131 \mathrm{~s}$ & -- \\
Unicyle [34,31] & $3619 \mathrm{~s}$ & $492 \mathrm{~s}$ & $516 \mathrm{~s}$ \\
\hline
\end{tabular}

Table 1 shows a comparison of functions Find_Pattern and Find_Pattern2, which shows that the new version highly improves the computation times. We can note that the new version is all the more efficient as the length of the patterns increases, and as obstacles cut the research tree of patterns. This is why we observe significant improvements on the examples of the DC-DC converter and the polynomial example, and not on the building ventilation example, which only requires patterns of length 2 , and presents no obstacle.

Table 2 shows of comparison of function Find_Pattern 2 with state-of-the-art tools SCOTS and PESSOA. On the example of the DC-DC converter, our algorithm manages to control the whole state-space $R=[1.55,2.15] \times$ $[1.0,1.4]$ in less than one second, while SCOTS and PESSOA only control a part of $R$, and with greater computation times. Note that these computation times vary with the number of discretization points used in both, but even with a very fine discretization, we never managed to control the whole box $R$. For the polynomial example, we manage to control the whole boxes $R_{1}$ and $R_{2}$, such as SCOTS and in a comparable amount of time. However, PESSOA does not support natively this kind of nonlinear systems. We compared our method on a last case study on which PESSOA and SCOTS perform well (see $[34,31]$ for details of this case study, and see Appendix for a simulation obtained using our method). For this case study, we have not obtained as good computations times as they have. This comes from the fact that this example requires a high number of switched modes, long patterns, as well as a high number of boxes to tile the state-space. Note that for this case study we used an automated pre-tiling of the state-space permitting to decompose the reachability problem in a sequence of reachability problems. This is in fact the most difficult case of application of our method. This reveals that our method is more adapted when either the number of switched modes of the length of patterns is not high (though it can be handled at the cost of high computa- tion times). Another advantage is that we do not require a homogeneous discretization of the state space. We can thus tile large parts of the state-space using only few boxes, and this often permits to consider much less symbolic states than with discretization methods, especially in high dimensions (see [22]).

\section{Conclusion}

We presented a method of control synthesis for nonlinear switched systems, based on a simple state-space bisection algorithm, and on validated simulation. The approach permits to deal with stability, reachability, safety and forbidden region constraints. Varying parameters and perturbations can be easily taken into account with interval analysis. The approach has been numerically validated on several examples taken from the literature, a linear one with constant parameters, and two nonlinear ones with varying perturbations. Our approach compares well with the state-of-the art tools SCOTS and PESSOA.

We would like to point out that the exponential complexity of the algorithms presented here, which is inherent to guaranteed methods, is not prohibitive. Two approaches have indeed been developed to overcome this exponential complexity. A first approach is the use of compositionality, which permits to split the system in two (or more) sub-systems, and to perform control synthesis on these sub-systems of lower dimensions. This approach has been successfully applied in [22] to a system of dimension 11, and we are currently working on applying this approach to the more general context of contract-based design [33]. A second approach is the use of Model Order Reduction, which allows to approximate the full-order system (1) with a reduced-order system, of lower dimension, on which it is possible to perform control synthesis. The bounding of the trajectory errors between the full-order and the reduced-order systems can be taken into account, so that the induced controller is guaranteed. This approach, described in [21], has been successfully applied on (space-discretized) partial differential equations, leading to systems of ODEs of dimension up to 100000 . The present work is a potential ground for the application of such methods to control of nonlinear partial differential equations, with the use of proper nonlinear model order reduction techniques.

\section{Acknowledgements}

This work is supported by Institut Farman (project SWITCHDESIGN), by the French National Research Agency through the "iCODE Institute project" funded by the IDEX Paris-Saclay, ANR-11-IDEX-0003-02, and by Labex DigiCosme (project ANR-11-LABEX-0045DIGICOSME). 


\section{References}

[1] Julien Alexandre dit Sandretto and Alexandre Chapoutot. Dynibex library. http://perso.ensta-paristech.fr/ chapoutot/dynibex/, 2015.

[2] Julien Alexandre dit Sandretto and Alexandre Chapoutot. Validated Solution of Initial Value Problem for Ordinary Differential Equations based on Explicit and Implicit RungeKutta Schemes. Research report, ENSTA ParisTech, 2015.

[3] Julien Alexandre dit Sandretto and Alexandre Chapoutot. Validated explicit and implicit runge-kutta methods. Reliable Computing, 22:79-103, 2016.

[4] Matthias Althoff. Reachability analysis of nonlinear systems using conservative polynomialization and non-convex sets. In Proceedings of the 16th international conference on Hybrid systems: computation and control, pages 173-182. ACM, 2013.

[5] A Giovanni Beccuti, Georgios Papafotiou, and Manfred Morari. Optimal control of the boost dc-dc converter. In Decision and Control, 2005 and 2005 European Control Conference. CDC-ECC'05. 44th IEEE Conference on, pages 4457-4462. IEEE, 2005.

[6] Olivier Bouissou, Alexandre Chapoutot, and Adel Djoudi. Enclosing temporal evolution of dynamical systems using numerical methods. In NASA Formal Methods, number 7871 in LNCS, pages 108-123. Springer, 2013.

[7] Olivier Bouissou and Matthieu Martel. GRKLib: a Guaranteed Runge Kutta Library. In Scientific Computing, Computer Arithmetic and Validated Numerics, 2006.

[8] Xin Chen, Erika Ábrahám, and Sriram Sankaranarayanan. Flow*: An analyzer for non-linear hybrid systems. In Computer Aided Verification, pages 258-263. Springer, 2013.

[9] A. Le Coent, J. A. dit Sandretto, A. Chapoutot, and L. Fribourg. Control of nonlinear switched systems based on validated simulation. In 2016 International Workshop on Symbolic and Numerical Methods for Reachability Analysis (SNR), pages 1-6, April 2016.

[10] L. H. de Figueiredo and J. Stolfi. Self-Validated Numerical Methods and Applications. Brazilian Mathematics Colloquium monographs. IMPA/CNPq, 1997.

[11] Tomáš Dzetkulič. Rigorous integration of non-linear ordinary differential equations in Chebyshev basis. Numerical Algorithms, 69(1):183-205, 2015.

[12] Andreas Eggers, Martin Fränzle, and Christian Herde. Sat modulo ode: A direct sat approach to hybrid systems. In Automated Technology for Verification and Analysis, pages 171-185. Springer, 2008.

[13] Goran Frehse, Colas Le Guernic, Alexandre Donzé, Scott Cotton, Rajarshi Ray, Olivier Lebeltel, Rodolfo Ripado, Antoine Girard, Thao Dang, and Oded Maler. Spaceex: Scalable verification of hybrid systems. In Computer Aided Verification, pages 379-395. Springer, 2011.

[14] Laurent Fribourg, Ulrich Kühne, and Romain Soulat. Finite controlled invariants for sampled switched systems. Formal Methods in System Design, 45(3):303-329, 2014.

[15] Karol Gajda, Małgorzata Jankowska, Andrzej Marciniak, and Barbara Szyszka. A survey of interval Runge-Kutta and multistep methods for solving the initial value problem. In Parallel Processing and Applied Mathematics, volume 4967 of LNCS, pages 1361-1371. Springer Berlin Heidelberg, 2008.

[16] Antoine Girard. Reachability of uncertain linear systems using zonotopes. In Hybrid Systems: Computation and Control, pages 291-305. Springer, 2005.
[17] Antoine Girard, Giordano Pola, and Paulo Tabuada. Approximately bisimilar symbolic models for incrementally stable switched systems. IEEE Transactions on Automatic Control, 55(1):116-126, 2010.

[18] Ernst Hairer, Syvert Paul Norsett, and Grehard Wanner. Solving Ordinary Differential Equations I: Nonstiff Problems. Springer-Verlag, 2nd edition, 2009.

[19] Fabian Immler. Verified reachability analysis of continuous systems. In Tools and Algorithms for the Construction and Analysis of Systems, pages 37-51. Springer, 2015.

[20] Ulrich Kühne and Romain Soulat. Minimator 1.0. https://bitbucket.org/ukuehne/minimator/overview, 2015.

[21] Adrien Le Coënt, Florian De Vuyst, Christian Rey, Ludovic Chamoin, and Laurent Fribourg. Control of mechanical systems using set based methods. International Journal of Dynamics and Control, pages 1-17, 2016.

[22] Adrien Le Coënt, Laurent Fribourg, Nicolas Markey, Florian De Vuyst, and Ludovic Chamoin. Distributed Synthesis of State-Dependent Switching Control, pages 119-133. Springer International Publishing, Cham, 2016.

[23] Daniel Liberzon. Switching in systems and control. Springer Science \& Business Media, 2012.

[24] Youdong Lin and Mark A. Stadtherr. Validated solutions of initial value problems for parametric odes. Appl. Numer. Math., 57(10):1145-1162, 2007.

[25] Jun Liu, Necmiye Ozay, Ufuk Topcu, and Richard M Murray. Synthesis of reactive switching protocols from temporal logic specifications. Automatic Control, IEEE Transactions on, 58(7):1771-1785, 2013.

[26] Manuel Mazo, Anna Davitian, and Paulo Tabuada. PESSOA: A Tool for Embedded Controller Synthesis, pages 566-569. Springer Berlin Heidelberg, Berlin, Heidelberg, 2010.

[27] Pierre-Jean Meyer. Invariance and symbolic control of cooperative systems for temperature regulation in intelligent buildings. Theses, Université Grenoble Alpes, September 2015.

[28] Pierre-Jean Meyer, Hosein Nazarpour, Antoine Girard, and Emmanuel Witrant. Experimental implementation of UFAD regulation based on robust controlled invariance. In Proceedings of the $13^{\text {th }}$ European Control Conference, pages 1468-1473, 2014.

[29] Ramon Moore. Interval Analysis. Prentice Hall, 1966.

[30] Nedialko S. Nedialkov, K. Jackson, and Georges Corliss. Validated solutions of initial value problems for ordinary differential equations. Appl. Math. and Comp., 105(1):21 68, 1999.

[31] Gunther Reissig, Alexander Weber, and Matthias Rungger. Feedback refinement relations for the synthesis of symbolic controllers. arXiv preprint arXiv:1503.03715, 2015.

[32] Matthias Rungger and Majid Zamani. Scots: A tool for the synthesis of symbolic controllers. In Proceedings of the 19th International Conference on Hybrid Systems: Computation and Control, HSCC '16, pages 99-104, New York, NY, USA, 2016. ACM.

[33] Alberto Sangiovanni-Vincentelli, Werner Damm, and Roberto Passerone. Taming dr. frankenstein: Contract-based design for cyber-physical systems. European journal of control, 18(3):217-238, 2012.

[34] Majid Zamani, Giordano Pola, Manuel Mazo, and Paulo Tabuada. Symbolic models for nonlinear control systems without stability assumptions. IEEE Transactions on Automatic Control, 57(7):1804-1809, 2012. 


\section{Appendix}

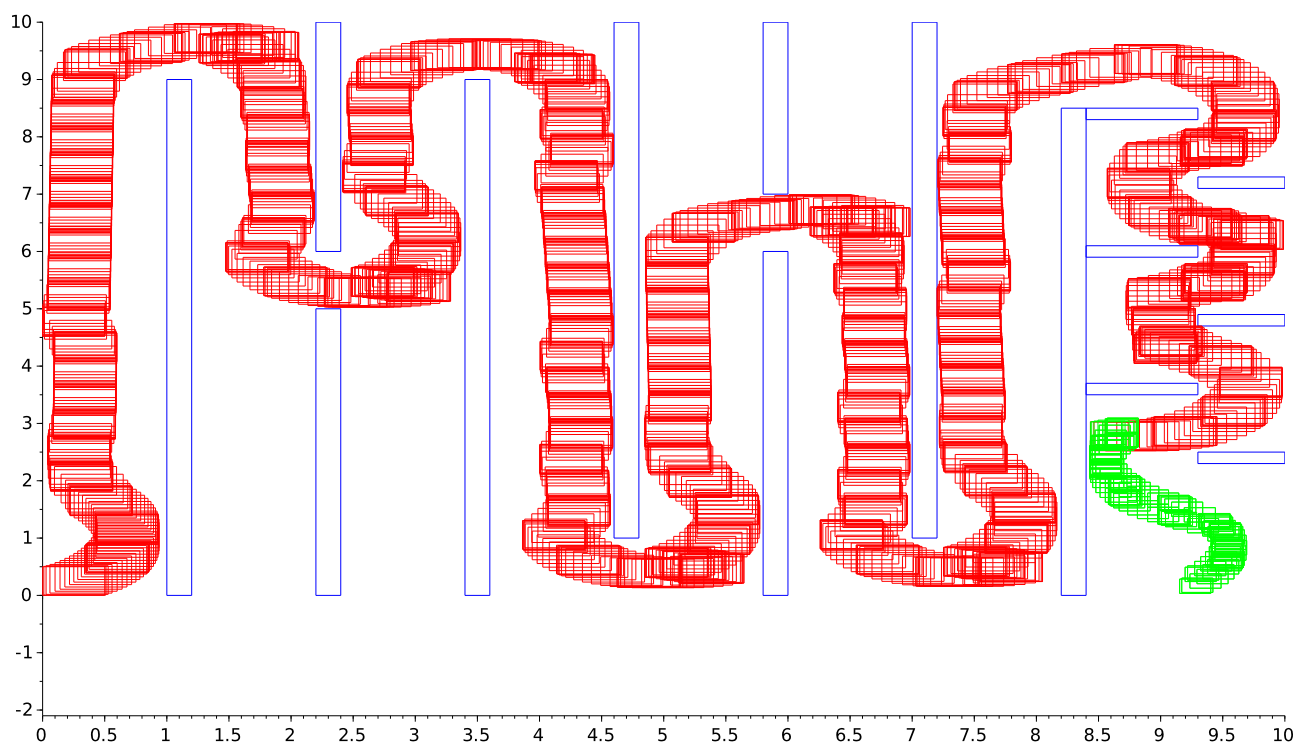

Fig. 10. Simulation of the unicycle example. 